九州大学学術情報リポジトリ

Kyushu University Institutional Repository

\title{
ON THE ORDERS OF MAX-MIN FUNCTIONALS
}

Hisano, Hiroshi

Nakamuragakuen Sanyou High School

Maruyama, Fumio

Department of Mathematics, Kyushu University

https://doi.org/10.5109/13403

出版情報: Bulletin of informatics and cybernetics. 23 (3/4)，pp.163-169，1989-03. Research Association of Statistical Sciences

バージョン :

権利関係 : 


\title{
ON THE ORDERS OF MAX-MIN FUNCTIONALS
}

\section{By}

\section{Hiroshi Hisano* and Fumio Maruyama*k}

\begin{abstract}
We construct a continuous function on $[0,1]^{n}$ that orders the set of Max-min functionals on $C\left([0,1]^{n}\right)$ according to an arbitrary given extended order of the natural partial order.
\end{abstract}

\section{Introduction}

Let $S_{1}, S_{2}, \cdots, S_{n}$ be compact spaces and let $\prod_{i=1}^{n} S_{i}$ be the direct product with the product topology. For a continuous real valued function $f\left(x_{1}, x_{2}, \cdots, x_{n}\right)$ on $\prod_{i=1}^{n} S_{i}$

$$
\operatorname{Max}_{x_{i}} f\left(x_{1}, x_{2}, \cdots, x_{n}\right) \text { and } \min _{x_{i}} f\left(x_{1}, x_{2}, \cdots, x_{n}\right) \quad(i=1,2, \cdots, n)
$$

are continuous functions of $(n-1)$-variables. Inductively, an $n$-length sequential maximizing or minimizing of a continuous function gives a mapping:

$$
C\left(\prod_{i=1}^{n} S_{i}\right) \rightarrow R
$$

where $C\left(\prod_{i=1}^{n} S_{i}\right)$ is the set of all real valued continuous functions on $\prod_{i=1}^{n} S_{i}$. We call this mapping a Max-min functional on $C\left(\prod_{i=1}^{n} S_{i}\right)$.

For a subset $T \subset C\left(\prod_{i=1}^{n} S_{i}\right)$, there might be no function of $T$ that mutually distinguishes all Max-min functionals. It depends on the cardinality and the topology of $S_{i}$ $(i=1,2, \cdots, n)$ and the subset $T$. The most well-kown non-trivial example is the following minimax theorem of von Neumann [2].

TheOREM (VON NEUMANN). Let

$$
\begin{aligned}
& S_{1}=\left\{\left(\xi_{1}, \xi_{2}, \cdots, \xi_{m}\right) \in[0,1]^{m} \mid \xi_{1}+\xi_{2}+\cdots+\xi_{m}=1\right\}, \\
& S_{2}=\left\{\left(\eta_{1}, \eta_{2}, \cdots, \eta_{n}\right) \in[0,1]^{n} \mid \eta_{1}+\eta_{2}+\cdots+\eta_{n}=1\right\},
\end{aligned}
$$

and let $T$ be the set of all bilinear forms. Then

$$
\forall f \in T \operatorname{Max}_{x_{1} \subseteq S_{1}} \min _{x_{2} \in S_{2}} f\left(x_{1}, x_{2}\right)=\min _{x_{2} \in S_{2}} \operatorname{Max}_{x_{1} \in S_{1}} f\left(x_{1}, x_{2}\right) .
$$

In this paper we consider the case $S_{1}=S_{2}=\cdots=S_{n}=[0,1]$ under its usual topology. In section 3 , where the role of each variable is fixed, we construct a polynomial of

* Nakamuragakuen Sanyou High School, Fukuoka, Japan.

** Department of mathematics, Kyushu University 33, Fukuoka 812, Japan. 
quite simple form whose degree is not greater than the number of Max-min functionals those are to be distinguished. A function determines an order on the set of Max-min functionals according to thier values at this function. In section 4 we construct a continuous function that determines the same arbitrary given order on the set of Max-min functionals as long as this order is consistent with the natural partial order.

As seen in [1] and [2] there is a close relation between Max-min functionals and the theory of games. The systematic structure of the constructed functions in this paper will give some information about the character of the games with which these Max-min functionals are concerned.

\section{Preliminaries}

We give some basic definitions and notations. For a fixed natural number $n$, $C\left([0,1]^{n}\right)$ denotes the set of all continuous real valued functions on $[0,1]^{n}$ under its usual product topology and $\Sigma$ denotes the set of all Max-min functionals on $C\left([0,1]^{n}\right)$. Let $r$ be the cardinality of $\Sigma$.

We will use $i, j, k$, as natural numbers less than or equal to $n$, $l, m, p, q$, as natural numbers less than or equal to $r$,

$d$, as an integer,

$x, y, t$, as real numbers in the closed interval $[0,1]$,

$\alpha$, as a non-empty subset of $\{1,2, \cdots, n\}$,

$\sigma, \mu$, as elements of $\Sigma$.

A Max-min functional $\sigma$ can be represented uniquely as

$$
\sigma=\prod_{k \leq m(\sigma)} \prod_{i \in A_{k}(\sigma)} \operatorname{Max}_{x_{i}} \prod_{j \in B_{k}(\sigma)} \min _{x_{j}},
$$

where

and

$$
A_{k}(\sigma), B_{k}(\sigma) \subset\{1,2, \cdots, n\}, A_{k}(\sigma) \cup B_{k}(\sigma) \neq \phi \quad(k=1,2, \cdots, m(\sigma)),
$$

$$
\bigcup_{k \leqq m(\sigma)}\left(A_{k}(\sigma) \cup B_{k}(\sigma)\right)=\{1,2, \cdots, n\} .
$$

Definition 2.1. $n(\sigma, i)=k$ iff $i \in A_{k}(\sigma) \cup B_{k}(\sigma)(i=1,2, \cdots, n)$.

DEFINITION 2.2 .

$$
\begin{aligned}
& \bar{X}(\sigma)=\bigcup_{k \leqq m(\sigma)} A_{k}(\sigma) . \\
& \underline{X}(\sigma)=\bigcup_{k \leqq m(\sigma)} B_{k}(\sigma) . \\
& \bar{X}(\sigma, i)=\bigcup_{k \leq n<\sigma, i)} A_{k}(\sigma) \quad \text { for } i \in \underline{X}(\sigma) . \\
& \underline{X}(\sigma, i)=\bigcup_{k<n(\sigma, i)} B_{k}(\sigma) \quad \text { for } i \in \bar{X}(\sigma) .
\end{aligned}
$$

Definition 2.3.

$$
\begin{aligned}
& S(\sigma)=\left\{\mu \mid \exists i \in \bar{X}(\sigma) \exists j \in \underline{X}(\mu)_{\cap} \underline{X}(\sigma, i) i \in \bar{X}(\mu, j)\right\} . \\
& T(\sigma)=\left\{\mu \mid \bar{X}(\sigma)_{\cap} \underline{X}(\mu) \neq \phi\right\} .
\end{aligned}
$$

For the natural order $<_{\Sigma}$ on $\Sigma$ (i.e. $\mu<_{\Sigma} \sigma$ iff $\mu \neq \sigma$ and $\forall f \in C\left([0,1]^{n}\right) \mu(f) \leqq \sigma(f)$ ) it is easy to observe that $\mu<_{\Sigma} \sigma$ implies $\mu \in S(\sigma) \cup T(\sigma)$. 


\section{Restricted Case}

In this section we fix $A \subset\{1,2, \cdots, n\}$ and consider $\Sigma_{A}=\{\sigma \mid \bar{X}(\sigma)=A\}$. If the cardinal number of $A=a$, then there is no loss of generality in assuming $A=\{1,2, \cdots, a\}$. Let $b=n-a, y_{k}=x_{a+k}, n^{\prime}(\sigma, k)=n(\sigma, a+k), \quad \beta(\sigma, k)=\bar{X}(\sigma, a+k) \quad(k=1,2, \cdots, b)$ and $B_{i}^{\prime}=\left\{j-a \mid j \in B_{i}\right\}$, where $B_{i} \subset B=\{a+1, a+2, \cdots, n\}$.

LEMMA 3.1.

$$
\prod_{l} \forall t_{l} \prod_{i} \exists x_{i}\left(\min _{l} \min _{i}\left|t_{l}-a^{-1} x_{i}\right|>\delta, \min _{\alpha \neq a^{\prime}}\left|a^{-1} \sum_{i \equiv \alpha} x_{i}-a^{-1} \sum_{i \equiv a^{\prime}} x_{i}\right|>2 \delta\right)
$$

for some positive $\delta$ that depends only on $n$.

PROOF. For each $i \leqq a$ choose $n_{i, l} \in\{1,2,3\}$ inductively so that the inequalities

$$
\left|t_{l}-a^{-1} \sum_{p \leqq l} n_{i, p} 5^{-r i-p}\right| \geqq a^{-1} 5^{-r i-l} \quad(l=1,2, \cdots, r)
$$

are satisfied. Put

$$
x_{i}=\sum_{l} n_{i, l} 5^{-r i-l} \quad(i=1,2, \cdots, a) .
$$

Then

$$
\begin{aligned}
\left|t_{l}-a^{-1} x_{i}\right| & >a^{-1} 5^{-r i-l} \cdot\left(1-5^{-1} \cdot 3 \cdot\left(1-5^{-1}\right)^{-1}\right) \\
& >a^{-1} 5^{-r i-l-1} \geqq a^{-1} 5^{-r a-r-1} \quad(i=1,2, \cdots, a)(l=1,2, \cdots, r) .
\end{aligned}
$$

If $\alpha \neq \alpha^{\prime}$ then $j \in\left(\alpha \backslash \alpha^{\prime}\right) \cup\left(\alpha^{\prime} \backslash \alpha\right)$ for some $j \leqq a$. It follows that

$$
\left|a^{-1} \sum_{i \in \alpha} x_{i}-a^{-1} \sum_{i \in \alpha^{\prime}} x_{i}\right|>a^{-1} 5^{-r j-1}>2 a^{-1} 5^{-r a-r-1} .
$$

The lemma is proved for $\delta \leqq a^{-1} 5^{-r a-r-1}$.

Lemma 3.2. Let $\sigma=\prod_{k \leq m(\sigma)} \prod_{i \in A_{k}(\sigma)} \operatorname{Max}_{x_{i}} \prod_{j \in B_{k}^{\prime}} \min _{y_{j}}$ and let

$$
f_{A}=\prod_{k \leq b} \prod_{\alpha}\left(c_{k, \alpha}\left(y_{k}-a^{-1} \sum_{j \in \alpha} x_{j}\right)^{2}+1\right),
$$

where $c_{k, \alpha}>2$ for $k \leqq b, \phi \neq \alpha \subset A$ and $c_{k, \alpha}<c_{k, \alpha^{\prime}}$ for $\alpha \varsubsetneqq \alpha^{\prime}\left(f_{A} \equiv 1\right.$ in the case $a=n$ or $a=0$ ). Then there exists a positive number $\varepsilon$ which depends only on $n$ such that for all $\sigma$

$$
\boldsymbol{\sigma}\left(f_{A}\right)>\varepsilon \prod_{k \leq b} c_{k, \hat{\beta}(\sigma, k)}^{-1} \prod_{\alpha} c_{k, \alpha^{\prime}}
$$

where $c_{k, \phi}=1$ in the case $\beta(\sigma, k)=\phi$.

PROOF. The inequalities

imply

$$
\min _{\alpha \neq \alpha^{\prime}}\left|a^{-1} \sum_{j \in \alpha} x_{j}-a^{-1} \sum_{j \in \alpha^{\prime}} x_{j}\right|>2 \delta, \quad\left|y_{k}-a^{-1} \sum_{j \in \alpha} x_{j}\right|<\delta
$$

$$
\forall \alpha^{\prime} \neq \alpha\left|y_{k}-a^{-1} \sum_{j=\alpha^{\prime}} x_{j}\right|>\delta
$$

Consequently, since $c_{k, \alpha} \leqq c_{k, \alpha^{\prime}}$ for $\alpha \subset \alpha^{\prime}$, if the inequalities

$$
\min _{k \leqq D} \min _{\alpha \Subset \beta(\sigma, k)}\left|y_{k}-a^{-1} \sum_{j=\alpha} x_{j}\right|>\delta, \quad \min _{\alpha \neq \alpha^{\prime}}\left|a^{-1} \sum_{j=\alpha} x_{j}-a^{-1} \sum_{j \leqslant a^{\prime}} x_{j}\right|>2 \delta
$$

are satisfied, then 


$$
f_{A}\left(x_{1}, x_{2}, \cdots, x_{n}\right)>\prod_{k \leqq b} c_{k, \hat{\beta}(\sigma, k)}^{-1} \prod_{\alpha}\left(\delta^{2} c_{k, \alpha}+1\right)>\delta^{2 b\left(2^{a}-1\right)} \prod_{k \leqq b} c_{k, \beta(\sigma, k)}^{-1} \prod_{\alpha} c_{k, \alpha} .
$$

But from Lemma 3.1

$$
\begin{gathered}
\prod_{k \leq m(\sigma)} \prod_{i \in A_{k}(\sigma)} \exists x_{i} \prod_{j \in B_{k}^{\prime}(\sigma)} \forall y_{j} \\
\left.\min _{k \leq b} \min _{\alpha=\xi_{j}(\sigma, k)}\left|y_{k}-a^{-1} \sum_{j=\alpha} x_{j}\right|>\delta, \quad \min _{\alpha \neq \alpha^{\prime}}\left|a^{-1} \sum_{j=\alpha} x_{j}-a^{-1} \sum_{j \in \alpha^{\prime}} x_{j}\right|>2 \delta\right)
\end{gathered}
$$

for some $\delta>0$. Therefore the lemma is proved for $\varepsilon \leqq \delta^{2 b\left(2^{a}-1\right)}$.

LEMNa 3.3.

$$
\sigma\left(f_{A}\right)<2^{b(2 a-1)} \prod_{k \leqq b} c_{k, \hat{\beta}(\sigma, k)}^{-1} \prod_{\alpha} c_{k, \alpha} .
$$

PROOF. For each $k \leqq b$, let $y_{k}=a^{-1} \sum_{j=\hat{s}(\sigma, k)} x_{j}$, then

$$
\begin{aligned}
f_{A}\left(x_{1}, x_{2}, \cdots, x_{n}\right) & <\prod_{k \leq b} c_{k, \beta(\sigma, k)}^{-1} \prod_{\alpha}\left(c_{k, \alpha}+1\right) \\
& <\prod_{k \leq b} c_{k, \beta(\sigma, k)}^{-1} \prod_{\alpha} 2 c_{k, \alpha}=2^{b\left(2^{\alpha}-1\right)} \prod_{k \leq b} c_{k, \beta(\sigma, k)}^{-1} \prod_{\alpha} c_{k, \alpha} .
\end{aligned}
$$

It follows that

$$
\sigma\left(f_{A}\right)<2^{b\left(2^{a}-1\right)} \prod_{k \leq b} c_{k, \hat{\zeta}(\sigma, k)}^{-1} \prod_{\alpha} c_{k, \alpha} .
$$

THEOREM 3.4. There exists a positive number $\varepsilon$ which depends only on $n$ such that if

$$
\operatorname{Max}_{\mu \neq \sigma} \min \left\{\prod_{k \leqq b} c_{k, \hat{\beta}(\sigma, k)} C_{k, \beta(\mu, k)}^{-1}, \prod_{k \leqq b} c_{k, \hat{\beta}(\mu, k)} C_{k, \beta(\sigma, k)}^{-1}\right\}<\varepsilon,
$$

then $\mu\left(f_{A}\right)<\sigma\left(f_{A}\right)$ follows from $\prod_{k \leq b} c_{k, \hat{\beta}(\sigma, k)}<\prod_{k \leqq b} c_{k, \beta(\mu, k)}$.

Proof. By Lemma 3.2, for some $\delta>0$,

$$
\delta \prod_{k \leqq b} c_{k, \beta(\sigma, k)}^{-1} \prod_{\alpha} c_{k, \alpha}<\sigma\left(f_{A}\right) .
$$

By Lemma 3.3

$$
\mu\left(f_{A}\right)<2^{b\left(2^{a}-1\right)} \prod_{k \leqq b} c_{k, \beta(\mu, k)}^{-1} \prod_{\alpha} c_{k, \alpha} .
$$

Then

$$
\prod_{k \leqq b} c_{k, \beta(\sigma, k)} c_{k, \beta(\mu, k)}^{-1}<2^{-b\left(2^{a}-1\right)} \delta \equiv \varepsilon
$$

implies

$$
\mu\left(f_{A}\right)<2^{b\left(2^{a}-1\right)} \prod_{k \leq b} c_{k, \beta(\mu, k)}^{-1} \prod_{\alpha} c_{k, \alpha}<\delta \prod_{k \leq b} c_{k, \beta(\sigma, k)}^{-1} \prod_{\alpha} c_{k, \alpha}<\sigma\left(f_{A}\right) .
$$

COROLlaRy 3.5. There exists a polynomial of degree $\left.\min \left\{2 b\left(2^{a}-1\right)\right\}, 2 a\left(2^{b}-1\right)\right\}$ such that $\sigma(f) \neq \mu(f)$ for any different $\sigma, \mu \subseteq \Sigma_{A}$.

PROOF. Let $f_{A}$ and $f_{B}$ be the polynomials in Theorem 3.4. Then the degrees of $f_{A}$ and $f_{B}$ are $2 b\left(2^{a}-1\right)$ and $2 a\left(2^{b}-1\right)$ respectively. For $\sigma \in \Sigma_{A}$,

$$
\begin{aligned}
\sigma\left(-f_{B}\right) & =\prod_{k \leq m(\sigma)} \prod_{i \in A(\sigma, k)} \operatorname{Max}_{x_{i}} \prod_{j \in B(\sigma, k)} \min _{x_{j}}\left(-f_{B}\right) \\
& =-\prod_{k \leq m(\sigma)} \prod_{i \subseteq A(\sigma, k)} \min _{x_{i}} \prod_{j \in B(\sigma, k)} \operatorname{Max}_{x_{j}}\left(f_{B}\right) .
\end{aligned}
$$

Consequently $f_{A}$ or $-f_{B}$ is the desired function. 
REMARK. Since there is no positive numbers $c_{1}, c_{2}, c_{3}$ such that $c_{1}<c_{2}, c_{2} c_{3}<c_{1} c_{3}$, no function $f$ of the type in Theorem 3.4 satisfies the inequalities

$$
\begin{aligned}
& \min _{y_{1}} \min _{y_{3}} \operatorname{Max}_{x} \min _{y_{2}}(f)<\min _{y_{2}} \min _{y_{3}} \operatorname{Max}_{x} \min _{y_{1}}(f), \\
& \min _{y_{2}} \operatorname{Max}_{x} \min _{y_{1}} \min _{y_{3}}(f)<\min _{y_{1}} \operatorname{Max}_{x} \min _{y_{2}} \min _{y_{3}}(f) .
\end{aligned}
$$

According to the general results in section 4 , there exists a continuous function satisfying these inequalities.

\section{General Case}

In this section we fix a linear order $<_{r}$ on $\Sigma$ which is an extension of the natural order $<_{\Sigma}$ and number the elements of $\Sigma$ according to $<_{r}$. I. e. $p<q$ iff $\sigma_{p}<_{r} \sigma_{q}$.

DEFINITION 4.1.

$$
h_{i}(x, y)=\operatorname{Max}\left\{\min \left\{2 \delta_{i}^{-1} \min _{d \leqq 2^{n_{i}}}|| 3 x-y\left|-d 2^{-n_{i}}\right|-1,1\right\}, 0\right\},
$$

where $n_{i}=i(n+3), \delta_{i}=2^{1-n_{i+1}}(i=1,2, \cdots, n)$.

$$
f_{<_{r}}\left(x_{1}, x_{2}, \cdots, x_{n}\right)=\underset{q<r}{\operatorname{Max}} \min _{p \leqslant q} \operatorname{Max}_{i \in \underline{X}\left(\sigma_{p}\right)} \operatorname{Max}_{j \in \bar{X}\left(\sigma_{p}, i\right)} l h_{j}\left(x_{i}, x_{j}\right)
$$

$\left(h_{0}(x) \equiv \operatorname{Max}_{j \equiv \dot{\phi}} h_{j}\left(x_{i}, x_{j}\right) \equiv \min \left\{2 x_{i}, 1\right\}\right.$ in the case $\left.\bar{X}\left(\sigma_{p}, i\right)=\phi\right)$.

LEMIMA 4.2.

$$
\forall i \prod_{j \neq i} \forall x_{j} \exists x_{i} \prod_{k} \forall y_{k} \min _{j \neq i} h_{i}\left(x_{j}, x_{i}\right)=\min _{k} h_{k}\left(x_{i}, y_{k}\right)=h_{0}\left(x_{i}\right)=1 .
$$

Proof. Let $\mu$ denote Lebesgue measure on $[0,1]$. For arbitrary $i, x$,

$$
\begin{aligned}
& \mu\left(\left\{y \mid h_{i}(x, y)=1\right\}\right)=\mu\left(\left\{y\left|\min _{d \leqq 2^{n_{i}}}\right||3 x-y|-d 2^{-n_{i}} \mid \geqq \delta_{i}\right\}\right) \\
= & \mu\left(\left\{y|| 3 x-y \mid \notin \bigcup_{d \leq 2^{n_{i}}}\left[d 2^{-n_{i}}-\delta_{i}, d 2^{-n_{i}}+\delta_{i}\right]\right\}\right) \\
\geqq & 1-2^{n_{i+1}} \delta_{i}=1-2^{i(n+3)+1-(i+1)(n+3)+1}=1-2^{-n-1} \geqq 1-4^{-1} n^{-1} .
\end{aligned}
$$

Consequently

$$
\forall i \prod_{j \neq i} \forall x_{j} \mu\left(\left\{y \mid \min _{j \neq i} h_{i}\left(x_{j}, y\right)=1\right\}\right) \geqq 1-(n-1)(4 n)^{-1}>4^{-1} \cdot 3,
$$

and so

$$
\forall i \prod_{j \neq i} \forall x_{j} \exists y>4^{-1} \cdot 3\left(\min _{j \neq i} h_{i}\left(x_{j}, y\right)=1\right) .
$$

But $y>4^{-1} \cdot 3$ implies

$$
\forall k \forall x h_{k}(y, x)=1, \quad h_{0}(y)=1 .
$$

Therefore the lemma follows from (1) and (2).

LEMMA 4.3. $\forall p \sigma_{p}\left(f_{<_{r}}\right) \geqq p-1$.

Proof. We claim

$$
\prod_{k \leqq m\left(\sigma_{p j}\right)} \prod_{i=A\left\langle\sigma_{p}, k\right)} \exists x_{i} \prod_{j=B\left\langle\sigma_{p}, k\right)} \forall x_{j} \forall q<p \quad g_{q}=1,
$$

where $g_{q}=\operatorname{Max}_{i \in X\left(\sigma_{q}\right)} \operatorname{Max}_{j \in \bar{X}\left(\sigma_{q}, i\right)} h_{j}\left(x_{i}, x_{j}\right)(q=1,2, \cdots, r-1)$. 
From Lemma 4.2

$$
\begin{aligned}
& \prod_{k \leq m\left(\sigma_{p}\right)} \prod_{i \in A\left(\sigma_{p}, k\right)} \exists x_{i} \prod_{j \in B\left(\sigma_{p}, k\right)} \forall x_{j} \\
& \left(\forall i \in \bar{X}\left(\sigma_{p}\right) \forall j \in \underline{X}\left(\sigma_{p}, i\right) \forall k \quad h_{i}\left(x_{j}, x_{i}\right)=h_{k}\left(x_{i}, x_{k}\right)=h_{0}\left(x_{i}\right)=1 ; .\right.
\end{aligned}
$$

Since $q<p$ implies $\sigma_{q} \in S\left(\sigma_{p}\right) \cup T\left(\sigma_{p}\right)$, there are two cases.

CASE 1. $\sigma_{q} \in S\left(\sigma_{p}\right)$.

Then

So

$$
\exists j \in \underline{X}\left(\sigma_{q}\right) \exists i \in \bar{X}\left(\sigma_{q}, j\right)\left(i \in \bar{X}\left(\sigma_{p}\right), j \in \underline{X}\left(\sigma_{p}, i\right)\right) .
$$

implies

$$
\forall i \in \bar{X}\left(\sigma_{p}\right) \forall j \in \underline{X}\left(\sigma_{p}, i\right) h_{i}\left(x_{j}, x_{i}\right)=1
$$

$$
\exists j \in \underline{X}\left(\sigma_{q}\right) \exists i \in \bar{X}\left(\sigma_{q}, j\right) h_{i}\left(x_{j}, x_{i}\right)=1 .
$$

CASE 2. $\sigma_{q} \in T\left(\sigma_{p}\right)$.

Then

So

$$
\exists i \in \bar{X}\left(\sigma_{p}\right)_{\cap} \underline{X}\left(\sigma_{q}\right) .
$$

implies

$$
\forall i \in \bar{X}\left(\sigma_{p}\right) \forall k h_{k}\left(x_{i}, x_{k}\right)=h_{0}\left(x_{i}\right)=1
$$

In both cases

$$
\exists i \in \underline{X}\left(\sigma_{q}\right) \operatorname{Max}_{j \in \bar{X}\left(\sigma_{q}, i\right)} h_{j}\left(x_{i}, x_{j}\right)=1 .
$$

$$
\forall i \in \bar{X}\left(\sigma_{p}\right) \forall j \in \underline{X}\left(\sigma_{p}, i\right) \forall k h_{i}\left(x_{j}, x_{i}\right)=h_{k}\left(x_{i}, x_{k}\right)=h_{0}\left(x_{i}\right)=1
$$

implies $g_{q}=1$.

Therefore (1) follows from (2). Then

Thus

$$
\prod_{k \leq m\left(\sigma_{p}\right)} \prod_{i \in A_{k}\left(\sigma_{p}, k\right)} \exists x_{i} \prod_{j \in B_{k}\left(\sigma_{p}, k\right)} \forall x_{j}\left(\min _{q \leq p-1}(p-1) g_{q}=p-1\right) .
$$

$$
\prod_{k \leq m\left(\sigma_{p}\right)} \prod_{i \in A_{k}\left(\sigma_{p}, k\right)} \exists x_{i} \prod_{j \in B_{k}\left(\sigma_{p}, k\right)} \forall x_{j}\left(\operatorname{Max}_{l<r} \min _{q \leq l} l g_{q} \geqq p-1\right) .
$$

LEMMA 4.4.

$$
\prod_{i} \forall x_{i} \exists x \operatorname{Max}_{i} h_{i}\left(x, x_{i}\right)=0 .
$$

PROOF. Since $\delta_{1}=2^{1-n_{2}}=2^{1-2(n+3)}<2^{-1}$ and $2^{-n_{1}}=2^{-(n+3)}<2^{-1}$, there exists a $d_{1} \leqq 2^{n_{1}}$ such that

$$
I_{1}=\left[x_{1}+d_{1} 2^{-n_{1}}-2^{-1} \delta_{1}, x_{1}+d_{1} 2^{-n_{1}}+2^{-1} \delta_{1}\right] \subset[0,1] .
$$

If $i>1$ and $I_{i-1}$ is a closed interval of length $\delta_{i-1}$ contained in $[0,1]$,

$$
\begin{aligned}
& \delta_{i}<2^{-1} \delta_{i-1} \text { and } 2^{-n_{i}}=2^{-1} \delta_{i-1} \text { imply } \\
& \exists d_{i} \leqq 2^{n_{i}}\left[x_{i}+d_{i} 2^{-n_{i}}-2^{-1} \delta_{i}, x_{i}+d_{i} 2^{-n_{i}}+2^{-1} \delta_{i}\right] \subset I_{i-1} .
\end{aligned}
$$

Thus, inductively, there exists a sequence of closed intervals

where for each $i$

$$
I_{n} \subset I_{n-1} \subset \cdots \subset I_{1} \subset[0,1],
$$

$$
\exists d_{i} \leqq 2^{n_{i}} I_{i}=\left[x_{i}+d_{i} 2^{-n_{i}}-2^{-1} \delta_{i}, x_{i}+d_{i} 2^{-n_{i}}+2^{-1} \delta_{\imath}\right] .
$$


If $3 x \in I_{n}=\bigcap_{i} I_{i} \subset[0,1]$, then $\prod_{i} \forall x_{i} \operatorname{Max}_{i} h_{i}\left(x, x_{i}\right)=0$.

LEMMA 4.5. $\forall p \sigma_{p}\left(f_{<_{r}}\right)=p-1$.

By Lemma 4.3, it suffices to prove $\forall p \sigma_{p}\left(f_{<_{r}}\right) \leqq p-1$.

Lemma 4.4 and $h_{0}(0)=0$ imply

Thus, for $p<r$,

$$
\forall \sigma \forall i \in \underline{X}(\sigma) \prod_{j \in \bar{X}(\sigma, i)} \forall x_{j} \exists x_{i} \operatorname{Max}_{j \in \bar{X}(\sigma, i)} h_{j}\left(x_{i}, x_{j}\right)=0 .
$$

$$
\prod_{k \leq m\left(\sigma_{p}\right)} \prod_{j \in A_{k}\left(\sigma_{p}\right)} \forall x_{j} \prod_{i \in B_{k}\left(\sigma_{p}\right)} \exists x_{i} \operatorname{Max}_{i \in X \underline{X}\left(\sigma_{p}\right)} \operatorname{Max}_{j \in \bar{X}\left(\sigma_{p}, i\right)} h_{j}\left(x_{i}, x_{j}\right)=0 .
$$

I. e. $\sigma_{p}\left(g_{p}\right)=0$. Consequently, for $p<r$,

$$
\begin{aligned}
\sigma_{p}\left(f_{<_{r}}\right) & =\sigma_{p}\left(\operatorname{Max}_{l<r} \min _{q \leq l} l g_{q}\right) \leqq \sigma_{p}\left(\operatorname{Max}\left\{p-1,(r-1) g_{p}\right\}\right) \\
& =\operatorname{Max}\left\{p-1,(r-1) \sigma_{p}\left(g_{p}\right)\right\}=p-1 .
\end{aligned}
$$

The inequality $\sigma_{r}\left(f_{<_{r}}\right) \leqq r-1$ is obvious.

THEOREM 4.6. Let $<_{s}$ be an extension of the natural order on $\Sigma$. Then for some $f \in C\left([0,1]^{n}\right)$ and arbitrary $\sigma, \mu \in \Sigma, \sigma<_{s} \mu$ implies $\sigma(f)<\mu(f)$.

Proof. Extend $<_{s}$ to a linear order $<_{r}$ on $\Sigma$. Then the theorem follows from Lemma 4.5 .

\section{Acknowledgement}

The authors would like to express their thanks to Professor Seiichi Iwamoto for leading them to this research.

\section{References}

[1] Iwamoto, S.: Dynamic Programming, Kyushu University Press, (1987) (Japanese).

[2] von Neumann, J.: Zur Theorie der Gesellschaftssiele, Mathematische Annalen, 100 (1928), 295-320.

Received February 18, 1988

Communicated by N. Furukawa 